\title{
Bâle - 2009 la Mecque de la médecine de famille
}

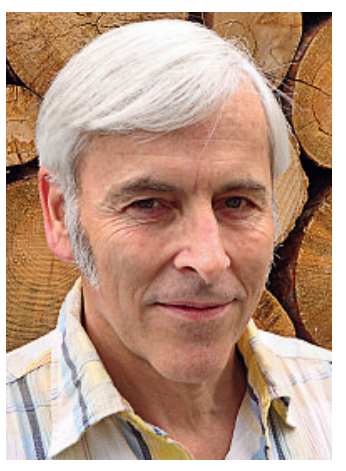

Peter Tschudi
«... the way a particular organism behaves will in part depend on its history, context and environment. This requires general practitioners to seek complexity and to accept uncertainty ... this means: of all clinical disciplines, general practice operates at the highest level of complexity, and consequent uncertainty ...» [1]

\section{Assister une fois dans sa vie à un congrès Wonca ...}

Après Londres, Amsterdam, Florence, Paris, Istanbul, le congrès Wonca-Europe [2] se déroulera à Bâle en 2009! Pour la première fois, la Suisse est le pays organisateur. On attend quelque 4000 médecins de famille en provenance de plus de 60 pays européens. Autant dire que c'est un événement que vous ne pouvez manquer sous aucun prétexte! Le comité d'organisation et le comité scientifique espèrent, qu'à l'instar du pèlerinage à la Mecque, les médecins de famille suisse et tous les amis de la médecine de premier recours ressentiront comme une nécessité absolue de participer au moins une fois dans leur vie à un congrès Wonca. Ce congrès qui se tiendra à Bâle du 16 au 19 septembre 2009 offre une occasion unique de mettre cette intention en pratique, pratiquement à deux pas de chez soi.

\section{Le thème du congrès:}

\section{"Complexité» et «Incertitude»}

L'action du médecin et à plus forte raison celle du médecin de famille se situe dans le champ de tension entre les «hard facts» linéaires de l'évidence scientifique et les «soft facts» complexes de l'expérience clinique du médecin de famille lors du traitement de patients individuels avec leur conception très complexe de la santé et de la maladie, de la vie et de la mort, avec leurs conditions de vie familiales et professionnelles personnelles, leurs croyances, leurs connaissances et leurs expériences, ou pour résumer, tout le contexte très personnel de leur existence.

La complexité et l'incertitude sont des composantes essentielles de la médecine de famille et caractérisent notre quotidien professionnel. Une gestion professionnelle de la complexité et de l'incertitude est une condition indispensable pour toute action médicale, notamment en médecine de famille.

Pourquoi la complexité et l'incertitude ont-elles une telle importance, précisément dans la médecine de famille? Certaines compétences de base des médecins de famille suffisent à l'illustrer:

- Premier interlocuteur pour toutes les maladies et troubles de la santé «non sélectionnés»

- Traitement de longue durée complet des malades chroniques et polymorbides

- Responsabilité pour l'individu et la société, avec par exemple gestion:

- de la peur de ...

- des nouvelles «maladies» (disease mongering)
- de la prévention et de la promotion de la santé

$-\ldots$

Vingt-quatre ateliers spécialisés avec une participation internationale traiteront et approfondiront le thème de la «complexité et de l'incertitude» dans le cadre du congrès.

\section{Le programme scientifique}

Les sept conférences principales (toutes traduites de manière simultanée en français et en allemand) porteront sur des thèmes d'actualité tels que la technologie de l'innovation, la nanomédecine, la neurobiologie, le génie génétique, les facteurs écologiques et environnementaux, toujours dans le contexte de la complexité de la médecine de premier recours. Chaque journée se conclura en outre par un «hot topic» («sujet chaud», p. ex. grippe porcine et vaccination HPV), à savoir un thème clinique de grande actualité ayant une incidence sur la pratique.

Le programme scientifique représente un total de 184 heures. Vous en trouverez le détail sur www. woncaeurope2009.org. Chaque participant(e) pourra très aisément constituer un programme sur mesure, adapté à ses besoins et ses intérêts, parmi les 130 heures d'ateliers, les 300 présentations et les 555 posters.

Le Congrès 2009 de la SSMG est intégré à cette manifestation et dix ateliers (qui se tiendront parallèlement en français et en allemand) seront spécialement proposés sur des thèmes importants (laboratoire, gynécologie, pédiatrie, psychiatrie) dans le cadre de la sauvegarde des acquis. Toutes les manifestations de la Wonca et de la SSMG sont ouvertes à l'ensemble des participants. Un droit d'inscription réduit est prévu pour les étudiant(e)s, les assistant(e)s et les jeunes médecins, pour que les jeunes et futurs collègues puissent assister nombreux à ce congrès.

\section{Un congrès qui n'est pas réservé aux seuls médecins de famille!}

Un congrès européen organisé en Suisse doit également être une vitrine pour la médecine suisse de premier recours et la présenter en conséquence. Aussi, le congrès s'adresse-t-il à toutes les personnes et associations intéressées par la médecine de famille. Nos partenaires issus de la politique, de l'assurance-maladie, des soins, les collègues spécialisés, notamment ceux qui travaillent dans les hôpitaux, sont cordialement invités à ces trois journées de médecine de famille intensive. Vous pouvez vous attendre à des échanges scientifiques passionnants! Profitez également de l'occasion pour vous immerger dans une atmosphère internationale.

Peter Tschudi* tions of General Practitioners/ Family Physicians».

Wonca ou «The World Organization of Family Doctors» signifie «World Organization of National Colleges, Acade- 